\title{
Analysis of Intelligent Transportation System Based on Fuzzy Algorithm
}

\author{
Shanshan Qu \\ Liaoning Police College, Liaoning, Dalian, China
}

Keywords: fuzzy algorithm; intelligent transportation; transportation system.

\begin{abstract}
As the economy is getting better and better, the masses are the basic consumption groups of the intelligent transportation system. For the vast number of manufacturers and operators, the masses are the resources they are trying to find. The research and development of high-tech products need time. It is necessary to adapt scientific research to the masses, so as to generate a continuous source of power and promote technological development. With the rapid development of network technology, the transmission of information through the network will certainly become a trend. Before the trip, first through the network to plan their own trip route; after the vehicle was stolen, the location of the vehicle was inquired through the positioning device. The new era of transmission of vehicle information through the network is taking the place of the old age of manpower scheduling step by step.
\end{abstract}

\section{Introduction}

With the rapid development of economy and the rapid growth of motor vehicles, traffic problems are becoming more and more serious. According to the current traffic conditions in our country, especially the traffic conditions in the metropolis and its vicinity, the overloading pressure are becoming more and more obvious. In China, the large and middle cities, represented by Guangzhou in Shanghai, Beijing, have shown a stronger demand for the application of intelligent transportation system. By drawing on the existing achievements and experiences of foreign ITS, the research on traffic light control system at home has broken the traditional time control time of the traffic light, adjusted the green time according to the change of the traffic flow, reached the maximum limit of the car release, reduced the traffic flow and alleviated the traffic congestion at the crossroads.

Fuzzy control belongs to the category of intelligent control in a broad sense. The application of fuzzy controller is the main reason that the fuzzy control system is different from the usual computer digital control system. It is the core of the operation of the fuzzy control system and is used in the mechanism of the fuzzy controller. Fuzzy rules, fuzzy decision methods and synthetic reasoning algorithms determine the main performance of a fuzzy control system.

Fuzzy controller is also known as fuzzy logic controller. Fuzzy controller is mainly influenced by the structure of fuzzy controller, the rules used, the method of fuzzy decision and the synthetic inference algorithm. Aiming at the traffic data of current traffic system can only be obtained by road monitor, the driver can not get the road information timely and accurately, and traffic managers are unable to control the overall situation and other defects, and form a traffic illness.

\section{Algorithm Design}

The membership function in fuzzy theory and fuzzy relation matrix describe the relationship between fault phenomenons and fault causes, and provide the scientific basis for the diagnosis of auto fault and repair method.

There are two domains, with the factors $U$ represent the fault phenomenon domains, evaluation set $V$ represents the failure domain, and the fault phenomenon membership derive fault membership. With a diagnosis of object has $n$ fault, may be expressed as $y_{1}, y_{2}, \cdots, y_{n}, m$ fault phenomenon caused by the failure, can be expressed as $x_{1}, x_{2}, \cdots, x_{\mathrm{m}}$. 
Therefore, fault fuzzy vector:

$Y=\left(y_{1}, y_{2}, \cdots, y_{i}, \cdots, y_{n}\right)$

Where, $y_{i}(i=1,2, \cdots, n)$ is the membership of fault reason $y_{i}$.

Fault phenomenon fuzzy vector:

$X=\left(x_{1}, x_{2}, \cdots, x_{i}, \cdots, x_{m}\right)$

Where, $x_{j}(j=1,2, \cdots, m)$ is the membership of fault phenomenon $x_{j}$.

$f=\frac{u_{0} \in A^{*}}{n}$

\section{Intelligent Algorithm Based on Fuzzy}

The control circuit (MCU) of intelligent transportation system is composed of single chip microcomputer, crystal oscillator circuit, reset circuit and key circuit. The schematic diagram of system is shown in Figure 2. Because of the restriction of the experimental environment, we use the button instead of the infrared tube to collect the traffic information in the laboratory. When the button is pressed, there is a vehicle passing through the test port in this direction. LED is used to indicate whether the system collects data. When the LED lamp is lit, the external interruption is opened, and the MCU begins to collect the port data; when the LED is extinguished, the interrupt is closed. An emergency button is set up in the system. If there is a special vehicle passing, then press the button and repeat it to resume normal state function.
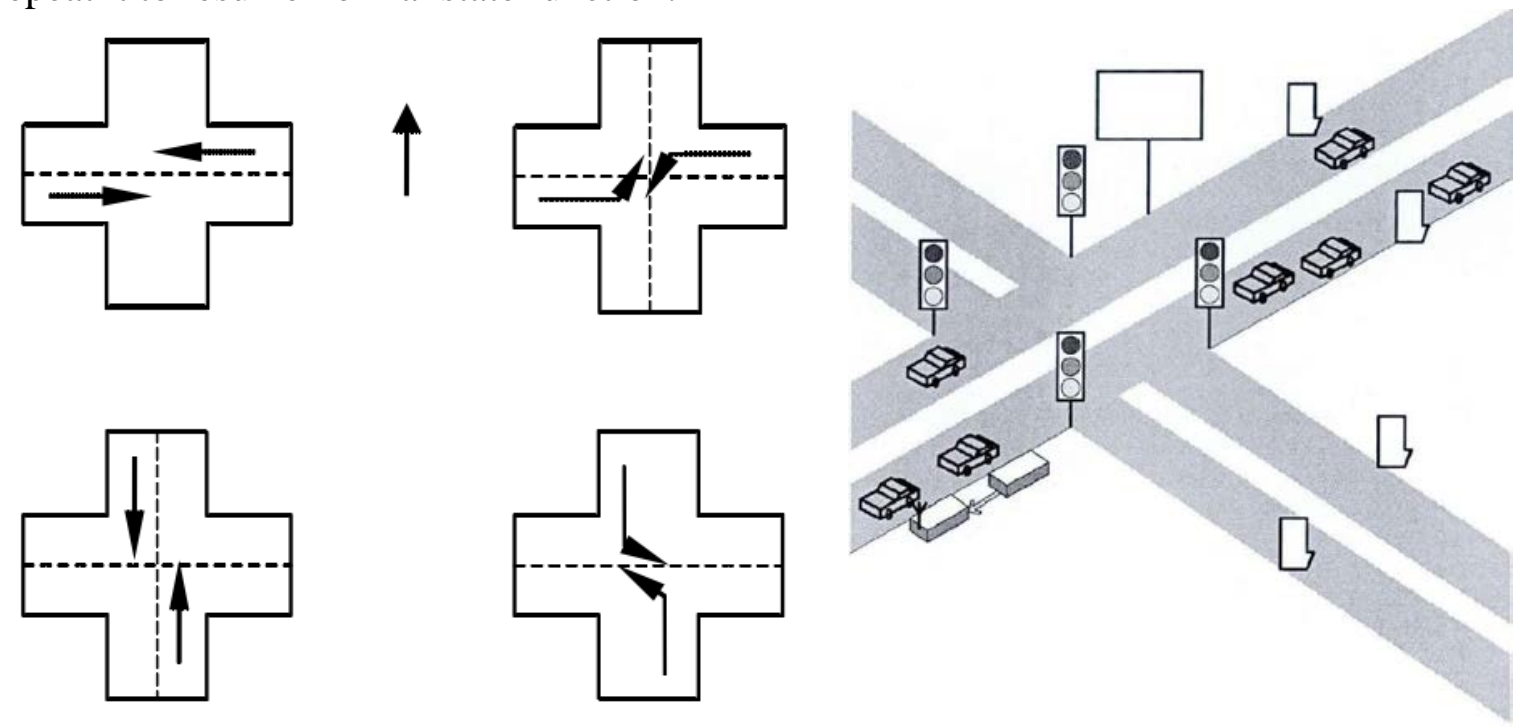

Fig. 1 Intelligent traffic control system plane simulation diagram

After the design and drawing of the PCB board and the electrical adjustment, the simulation model of the system is built. The building of the model is based on the four phase and eight lane design. When the car is detected, the level signal is transmitted to the single chip microcomputer. Special emergency vehicles are used to complete actual interruptions. Each lane is carried out by three photoelectric sensors. Considering the limited port of single chip microcomputer, 74LS138 decoder is used to select 74LS245, one end of 74LS245 is connected with photoelectric sensor, the other end is connected with single chip microcomputer, and each photoelectric sensor is scanned and detected by single chip microcomputer.

\{Void External_interruption_0(void)

TRISB $=0 \mathrm{x} 01$;

PORTBbits. RBO=1;

INTCONbits.RBIE $=0$;

INTCON2bits.INTEDGO=0;

INTCONbits.INTOIF=0; 
INTCONbits.INTOIE=1;

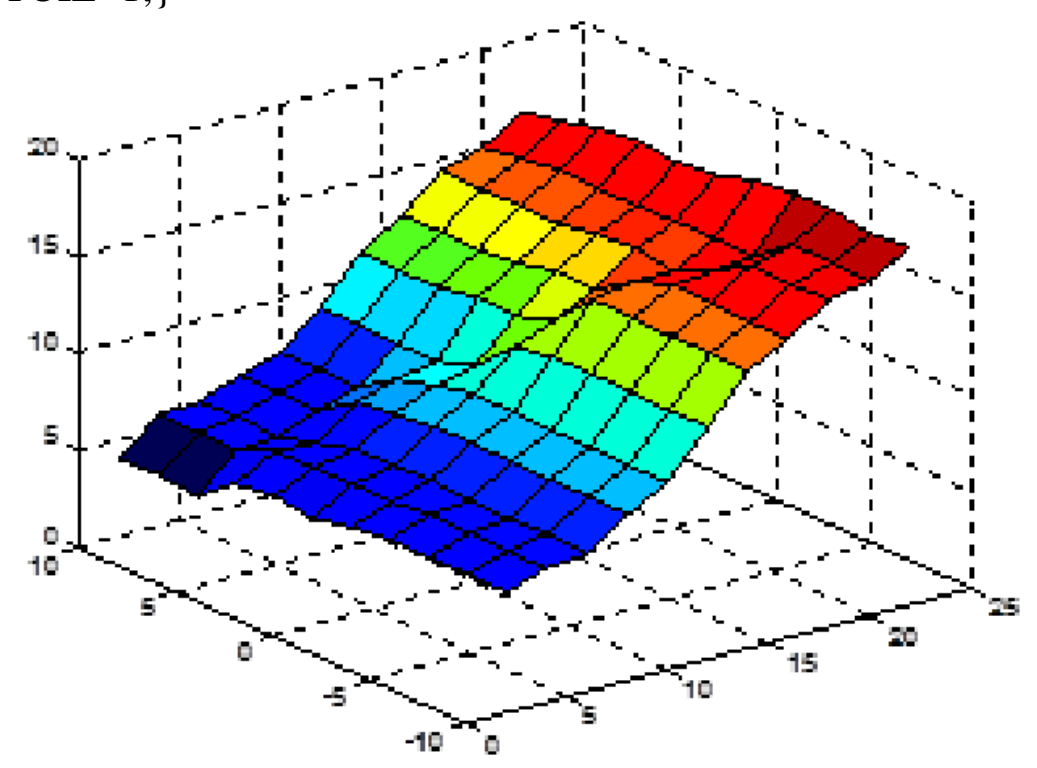

Fig. 2 Fuzzy control plane diagram

After completing the construction of the intelligent traffic system hardware platform, corresponding software design is also needed to achieve the control function of the entire traffic light system. Whether the software design is reasonable or not determines whether the entire system can achieve the corresponding function and whether it has a high degree of safety and reliability. Therefore, a modular design method is used to design the control system program. By decomposing all the functions into several simpler sub-functions, each relatively independent sub-function module is responsible for completing each sub-function and calling the sub-program.

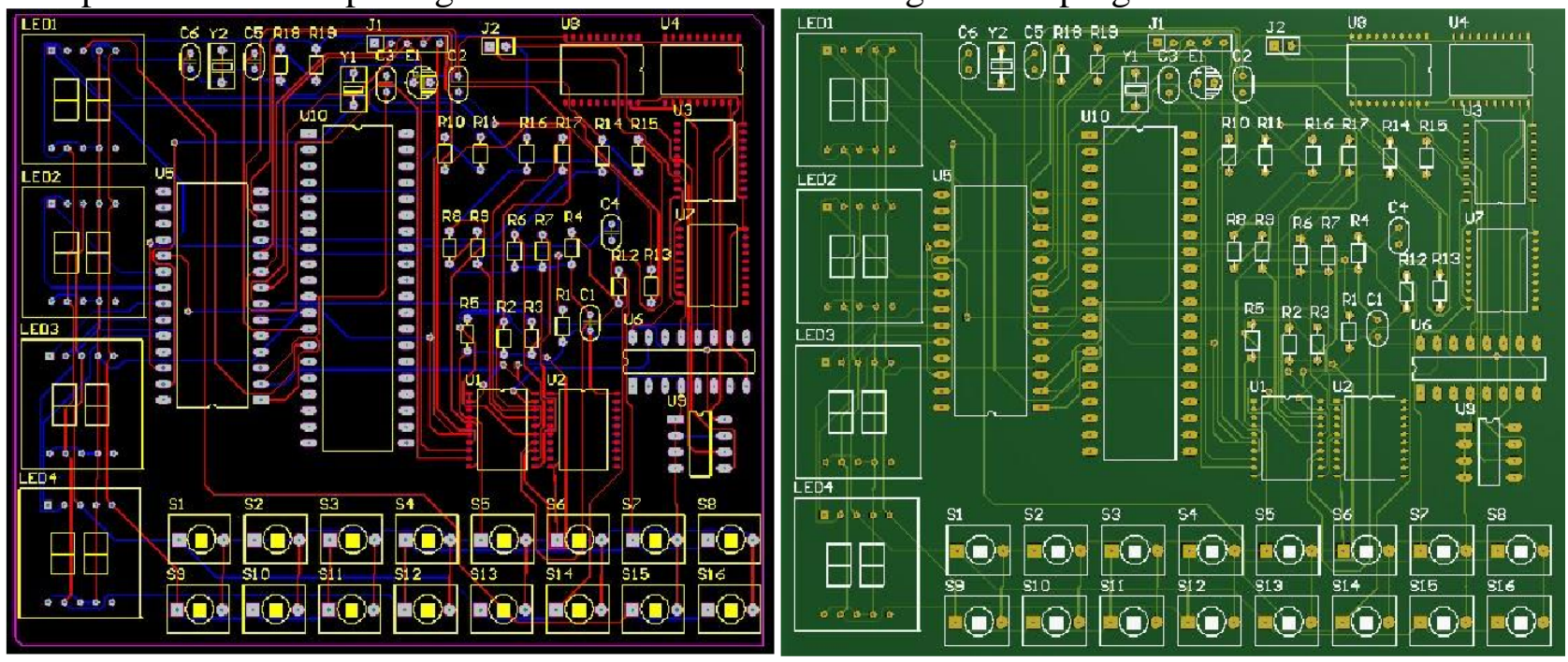

Fig. 3 Control circuit of Intelligent transportation system

The system working software is programmed in the c language environment. The program interface is shown in Figure 2. The main contents include: control of the main program, control subroutines and interrupt program modules. First, the main program of the system initializes the system, writes some keywords into each module of the system, and then starts a continuous key scan process, to see how many vehicles pass through the intersection within a specified time, the flow chart of the main program. As shown in Figure 3. Initialization functions include LCD, timers, external interrupts, and serial port interrupt initialization, as well as enabling interrupts and setting interrupt priority levels.

The fuzzy controller is implemented using a digital computer and has three important functions: First, the system's deviation is converted from a digital quantity to a fuzzy quantity. This part is 
completed by the fuzzification process and the knowledge base; then the fuzzy quantity is blurred by the given rules. Reasoning; Finally, the precise output of the fuzzy output is converted into an accurate digital quantity that can be accepted by the actual lesson preparation system. Therefore, the design of the fuzzy controller includes the contents of the following four divisions: fuzzification process, knowledge base establishment, fuzzy inference process, and control Clear calculations.

The basic process of the automatic control system is to compare the input value of a certain time with the control value, and then make the optimal control of the system by comparing the difference obtained by the comparison. In the fuzzy controller, the reference value in the control rule library is generally expressed by integers, while the input value is accurate. Therefore, in order to match the data format between them, the input variables need to be fuzzed. The fuzzification process is completed mainly: the value of the input variable is measured, and the input amount of the digital representation is converted to the number of a certain limit code expressed by the general language value.

The knowledge base contains two parts: database and rule library, in which the database represents the definition of system discretization, quantification, normalization, and membership function; The rule library uses fuzzy language to establish a complete set of query list libraries based on fuzzy control rules, usually using Matlab language. The system gives a combination of control rules described by language variables and generated by experts or self-learning according to control purposes and control rules, such as the selection of state variables, the rotation of control quantities, the selection of rule types, and the determination of rule purposes.

\section{Summary}

The intelligent traffic control method based on fuzzy control is studied, and the role of fuzzy control algorithm in traffic control system is analyzed. This paper focuses on a two-level fuzzy control theory algorithm, and the first-level fuzzy control based on the average waiting time of vehicles in other phases. Queue length makes a fuzzy decision. This method can make up for the current shortage of the traffic system in terms of hardware, reduce the queue phenomenon of the vehicle, and improve the traffic speed of the vehicle.

\section{References}

[1] Zhai J, Cao Y, Chen Y. Semantic information retrieval based on fuzzy ontology for intelligent transportation systems (IEEE International Conference on Systems, Man and Cybernetics. IEEE, 2009) p.2321-2326.

[2] Sun X. Fuzzy evaluation on archives digitization level based on improved algorithm (International Conference on Power Electronics and Intelligent Transportation System. IEEE, 2010) p.17-20.

[3] Bojan T M, Kumar U R, Bojan V M. An internet of things based intelligent transportation system (IEEE International Conference on Vehicular Electronics and Safety. IEEE), (2014), p. 174-179.

[4] Yan Y, Xu C. A Development Analysis of China's Intelligent Transportation System (Green Computing and Communications. IEEE), (2013), p. 1072-1076.

[5] Bo X, Shiru Q U. Intelligent Vehicle's Path Tracking Based on Fuzzy Control (Journal of Transportation Systems Engineering \& Information Technology, 2010), 10(2), p.70-75.

[6] Ma W G. Research on Design and Algorithm of Fuzzy Controller in Intelligence Transportation System (Intelligent Control and Automation, 2006. WCICA 2006. The Sixth World Congress on. IEEE, 2006) p.3848-3851. 